九州大学学術情報リポジトリ

Kyushu University Institutional Repository

\title{
Antioxidant therapy attenuates myocardial telomerase activity reduction in superoxide dismutase-deficient mice
}

Makino, Naoki

Division of Molecular and Clinical Gerontology, Department of Molecular and Cellular Biology, Medical Institute of Bioregulation, Kyushu University

Maeda, Toyok i

Division of Molecular and Clinical Gerontology, Department of Molecular and Cellular Biology, Medical Institute of Bioregulation, Kyushu University

Oyama, Jun-ichi

Division of Molecular and Clinical Gerontology, Department of Molecular and Cellular Biology, Medical Institute of Bioregulation, Kyushu University

Sasaki, Makoto

Division of Molecular and Clinical Gerontology, Department of Molecular and Cellular Biology, Medical Institute of Bioregulation, Kyushu University

他

http://hdl. handle. net/2324/25520

出版情報：Journal of Molecular and Cellular Cardiology. 50 (4)，pp.670-677，2011-04. Elsevier バージョン：

権利関係: (C) 2011 Elsevier Ltd. 


\section{Antioxidant Therapy Attenuates Myocardial Telomerase Activity Reduction in Superoxide Dismutase-Deficient Mice}

Naoki Makino ${ }^{1}$, Jun-ichi Oyama ${ }^{1}$, Toyoki Maeda ${ }^{1}$, Makoto Sasaki ${ }^{1}$, Yoshihiro Higuchi ${ }^{1}$, Koji Mimori ${ }^{2}$, Takahiko Shimizu ${ }^{3}$

${ }^{1}$ Division of Molecular and Clinical Gerontology, Department of Molecular and Cellular Biology, ${ }^{2}$ Department of Surgery Medical Institute of Bioregulation, Kyushu University, Beppu, JAPAN ${ }^{3}$ Molecular Gerontology, Tokyo Metropolitan Institute of Gerontology, Itabashi-ku, Tokyo 173-0015, Japan

Contact information:

Naoki Makino M.D., Ph.D.

Division of Molecular and Clinical Gerontology, Department of Molecular and Cellular Biology, Medical Institute of Bioregulation, Kyushu University, 4546 Tsurumihara, Beppu, 874-0838 Japan

Ph: 81-977 27 1681, Fax: 81-977 271682

E-mail: makinon@beppu.kyushu-u.ac.jp

\section{Abstract}

Oxidative stress plays a pathological role in the development of heart failure.

This study examined telomere biology in heart/muscle-specific manganese superoxide dismutase -deficient mice (H/M-SOD2 $\left.{ }^{-/}\right)$, which develop progressive 
congestive heart failure and exhibit pathology typical of dilated cardiomyopathy. EUK-8 (25mg/kg/day), a superoxide dismutase and catalase mimetic, was administered to $\mathrm{H} / \mathrm{M}-\mathrm{SOD} 2^{-/}$mice for four weeks beginning at 8 weeks of age. Telomere length, telomerase activity, telomere-associated proteins, and cell death signals were assessed in hearts from control wild-type mice (H/M-Sod2 lox/ ${ }^{l o x}$ ) and $\mathrm{H} / \mathrm{M}-\mathrm{SOD} 2^{-1-}$ mice either treated or untreated with EUK-8. While cardiac function was unchanged in these experimental mice, the end diastolic dimension in $\mathrm{H} / \mathrm{M}^{-S O D} 2^{-/}$mice was notably dilated and could be significantly reduced by EUK-8 treatment. At the end of the study, no shortening of telomere length was observed in heart tissues from all mice tested, but telomerase activity was decreased in heart tissue from $\mathrm{H} / \mathrm{M}-\mathrm{SOD} 2^{-/-}$mice compared to control mice. Protein expressions for telomerase reverse transcriptase and telomere repeat binding factor 2 were also downregulated in $\mathrm{H} / \mathrm{M}-S O D 2^{-/}$heart tissue as was expression of phospho-Akt, insulin-like growth factor, and endothelial nitric oxide synthase. Expression levels of Sirt1, a life-span modulator, were enhanced while FoxO3a was depressed in $\mathrm{H} / \mathrm{M}-\mathrm{SOD} 2^{-1-}$ hearts. All of the changes seen in H/M-SOD2 $2^{-1}$ heart tissue could be inhibited by EUK-8 treatment. Taken together, the results suggest that oxidant stress might affect myocardial telomerase activity and telomere-associated proteins. Telomerase may therefore play a pivotal role in antioxidant defense mechanisms, and may be useful as a novel therapeutic tool for treating human heart failure.

Key words: cardiomyopathy, free radical, oxidative stress, superoxide dismutase, telomere, telomerase, heart failure 


\section{Introduction}

Several clinical studies support the hypothesis that reactive oxygen species (ROS) play an important role in the pathogenesis of heart failure (1). Mitochondria generate ROS during cellular aerobic metabolism, but production of ROS, including superoxide anions $\left(\mathrm{O}_{2}^{-}\right)$and hydrogen peroxide $\left(\mathrm{H}_{2} \mathrm{O}_{2}\right)$, may be associated with increased morbidity. Cells synthesize protective proteins, such as mitochondrial manganese-superoxide dismutase (Mn-SOD), glutathione peroxidase, and catalase, in addition to non-enzymatic antioxidants, to guard against the effects of ROS. Mn-SOD is a superoxide scavenger enzyme located in the mitochondrial matrix that protects cells from mitochondrial ROS by detoxifying $\mathrm{O}_{2}$ radicals via dismutation to yield $\mathrm{H}_{2} \mathrm{O}_{2}$ and $\mathrm{H}_{2} \mathrm{O}$.

Mn-SOD-deficient mice have been independently generated by deleting different segments of the Sod2 gene $(3,4)$. Heart/muscle-specific Mn-SOD-deficient $\left(\mathrm{H} / \mathrm{M}-\mathrm{Sod} 2^{-1} ; \mathrm{KO}\right)$ mice are a model of dilated cardiomyopathy (DCM) involving an excess generation of ROS by mitochondria $(5,6)$. These findings suggest that Mn-SOD plays an important role in susceptibility to DCM. The synthetic salen-manganese complex, EUK-8, possesses both SOD and catalase activity and is a potent SOD/catalase mimetic and antioxidant $(7,8)$. EUK-8 can dismutate $\mathrm{O}_{2}{ }^{-}$to $\mathrm{H}_{2} \mathrm{O}_{2}$ and then catalyze $\mathrm{H}_{2} \mathrm{O}_{2}$ breakdown to $\mathrm{O}_{2}$ and $\mathrm{H}_{2} \mathrm{O}$. In vivo studies showed that EUK-8 abrogates cardiac mitochondrial dysfunction (9) and delays aging (10). Interestingly, EUK-8 treatment has been found to extend the lifespan of wild-type nematodes (11) and Sod2-null mutant mice (12). Telomeres are tandem repeats of the DNA sequence TTAGGG, which extends 
over $6-15 \mathrm{~kb}$ at the ends of eukaryotic chromosomes and are necessary for both successful DNA replication and chromosomal integrity (13). Telomeres in somatic human cells shorten by $30-200$ bp upon each cell division, and once they shorten to a critical length, cells are triggered to enter replicative senescence, involving an irreversible cell-cycle block in $\mathrm{G}_{0} / \mathrm{G}_{1}(14)$, in which cell function is altered (15) and cells are more likely to undergo apoptosis if exposed to increased oxidative insult (16). Telomerase and telomerase reverse transcriptase (TERT) have been suggested to regulate cardiac muscle cell growth and survival (17). Mice lacking telomerase displayed cardiac dysfunction, increased expression of p53, and increased apoptosis (18). However, the role of oxidant stress in telomere biology has not been clarified in heart failure.

Because both oxidative stress and telomerase dysfunction appear to be involved in the development of heart failure, the present study was undertaken to examine the role of telomere biology in heart failure. To do this, we examined telomere length and telomerase activity in MnSOD-deficient mice and the effects of the antioxidant SOD and catalase mimetic EUK-8

\section{Methods}

\section{Animals}

We used KO mice, produced by crossbreeding Mn-SOD ${ }^{\text {loxllox }}$ mice with muscle creatine kinase-Cre transgenic mice using in vitro fertilization techniques. Genotyping was performed by the tail DNA polymerase chain reaction, as described previously $(5,8)$. Wild-type mice $\left(H / M-S o d 2^{\text {lox/lox}}\right)$ were used as a control. All mice were housed in plastic cages (one animal/cage) in a pathogen-free barrier facility and kept under a 12-h light/dark cycle. Animal 
experiments conformed to the Guide for the Use and Care of Laboratory Animals (NIH Pub. No. 85-23, revised 1996) issued by the U.S. National Institutes of Health. The experiments were conducted in accordance with the Kyushu University Guide for the Treatment of Laboratory Animals.

\section{Antioxidant treatment}

Administration of EUK-8 (Calbiochem, San Diego, CA, USA) suspended in saline at $1.5 \mathrm{mg} / \mathrm{ml}$ was injected intraperitoneally ( $25 \mathrm{mg} / \mathrm{kg}$ body weight) into the mice 3 times a week (7). The administration began when the animals were 8 weeks of age and was continued until 12 weeks of age with EUK-8 or an equal volume of vehicle (phosphate-buffered saline) injected for the duration of the study.

\section{Genomic DNA extraction from mice tissue}

Mouse tissue samples were lysed by incubation at $55^{\circ} \mathrm{C}$ for 48 hours in $200 \mu \mathrm{L}$ lysis buffer containing $10 \mathrm{mM}$ Tris/HCl (pH 8.0), $0.1 \mathrm{mM}$ EDTA ( $\mathrm{pH} 8.0), 2 \%$ sodium dodecyl sulfate (SDS), and $500 \mu \mathrm{g} / \mathrm{mL}$ protease $\mathrm{K}$ (Roche Diagnostic, Tokyo, Japan). Genomic DNA extraction was performed using the DNeasy Tissue Kit (Qiagen K.K., Tokyo, Japan) according to the manufacturer's recommendations, as described previously (19).

\section{Western blot and other analyses}

Left ventricular tissue was homogenized with $500 \mu$ lysis buffer (100 mM Tris pH $6.8,4 \%$ SDS, $20 \%$ glycerol containing the protease inhibitor $M$ phenylmethanesulfonyl fluoride, $0.1 \mathrm{mM}$, leupeptin, $0.5 \mu \mathrm{l}$, and aprotinin, $0.5 \mu \mathrm{l}$. Gel electrophoresis was used to separate $50 \mu \mathrm{g}$ protein on a $10 \%$ SDS-polyacrylamide gel. Proteins were transferred to nitrocellulose membranes 
(162-0112, Bio-Rad Laboratories, Hercules, California) blocked with 5\% dry milk or blocking solution for Western blot (Roche) and exposed to rabbit polyclonal immunoglobulin G (IgG) TRF2 (H-300: sc-9143, Santa Cruz Biotechnology, Santa Cruz, California; dilution 1:200), mouse monoclonal IgG p16 (F-12: sc-1661, Santa Cruz Biotechnology; dilution 1:250), rabbit polyclonal IgG anti-p53 (FL-393: sc-6243, Santa Cruz Biotechnology; dilution 1:500 in dry milk 1\%), mouse monoclonal IgG Chk2 (A-11: sc-17747, Santa Cruz Biotechnology; dilution 1:1,000 in dry milk 1\%), rabbit polyclonal IgG anti-p-Akt (Ser473) (\#9271, Cell Signaling Technology, Danvers, Massachusetts; dilution 1:1,500), mouse IgG anti-p-eNOS (pS1177) (\#612392, Becton Dickinson; dilution 1:1,000), Mn-SOD (1:10,000; catalog number SOD-111; StressGen, Victoria, Canada), silent information regulator 1 (Sirt1), and mouse monoclonal IgG glyceraldehyde-3-phosphate dehydrogenase (GAPDH) (6C5: sc-32233, Santa Cruz Biotechnology; dilution 1:1,000). For analysis of TERT, immunoprecipitation was performed using polyclonal IgG anti-TERT (H-231: sc-7212, Santa Cruz Biotechnology) and agarose-A protein goat anti-rabbit IgG (Sigma-Aldrich). Antibodies Sirt1 and forkhead transcription factors 3a (FoxO3a) were purchased from Santa Cruz Biotechnology. Immunodetection was performed using goat anti-rabbit IgG (Sigma-Aldrich) and goat anti-mouse (170-6516, Bio-Rad) secondary antibodies (1:4,000 dilution), and an enhanced chemiluminescence kit (Amersham Biosciences).

\section{Telomerase activity}

Telomerase activity was examined using a modified telomerase repeat amplification protocol method (19) with TeloChaser (Toyobo, Osaka, Japan) 
according to the manufacturer's instructions. The intensities of the bands were quantified with ImageJ $(\mathrm{NIH})$. For each species, telomerase activity was analyzed in seven tissue types from three to six animals. The assays were repeated at least twice for each individual animal in order to ensure reproducibility. A human cancer cell line overexpressing telomerase was used as a reference in each assay.

\section{Telomere Length Measurement}

Telomere length was analyzed by Southern blot using the mean telomere refraction fragment (TRF) method, as previously described (20). Briefly, genomic DNA was extracted from heart or liver and digested with a mixture of Alul, Haelll, Rsal, and Hinfl restriction enzymes that do not cut within the telomeric repeat sequence. The resulting fragments were separated using pulse field gel electrophoresis, and hybridized with a 500-bp-long (TTAGGG)n digoxigenin (dig)-labeled probe specific for telomeric repeats. The blotted membranes were incubated with anti-dig-specific antibody conjugated with alkaline phosphatase. The mean TRF was determined using a telomere percentage analysis with four intervals of length as defined by a molecular weight standard.

\section{Immunohistochemistry}

For histological analysis, heart tissues ( $\mathrm{n}=6$ in each group) were immersed in $10 \%$ buffered formalin. Fixed tissues were dehydrated, embedded in paraffin, sectioned into $4 \mu \mathrm{m}$ slices, and stained with hematoxylin and eosin (H\&E) or Masson's trichrome stain. As a marker of oxidant stress, 8-hydroxydeoxyguanosine (8-oxodG) was measured using a previously reported 
procedure (21). Samples were treated with $10 \mu \mathrm{g} / \mathrm{ml}$ Proteinase $\mathrm{K}$ in phosphate-buffered saline (PBS) (0.05 M phosphate, $0.15 \mathrm{M} \mathrm{NaCl}, \mathrm{pH} 7.4)$ with $1 \%$ bovine serum albumin (BSA-50, Rockland, USA) for $40 \mathrm{~min}$ at $37^{\circ} \mathrm{C}$, and incubated in 5\% skim milk in PBS for $2 \mathrm{~h}$. The sections were next incubated with monoclonal anti-8-oxodG antibody (N45.1 Gentaur MOG-020P) (1:75) for $2 \mathrm{~h}$ at room temperature and overnight at $4^{\circ} \mathrm{C}$, and then sequentially exposed to biotin-labeled horse anti-mouse IgG and ABC complex (Vectastain ABC elite kit, Vector Laboratories, USA). After thorough washing, Alexa Fluor 660 goat anti-rabbit IgG $(H+L)$ (1:500 dilution, Molecular Probes Inc., Eugene, OR, USA) secondary antibodies were applied for an additional 60 min at $37{ }^{\circ} \mathrm{C}$. Nuclei were stained with 4',6-diamino-2-phenylindole (DAPI) where indicated. Fluorescence imaging was performed with an Olympus BX51 (Olympus) equipped for epi-fluorescence microscopy with a CCD camera, and $8 \mathrm{OH}-\mathrm{dG}$ signals were quantified and the data expressed as particle number/area fraction.

\section{Transthoracic echocardiography}

Noninvasive echocardiographic measurements were performed using the LOGIQ 400PRO system (GE Medical Systems, Milwaukee, WI) equipped with a $10-12-\mathrm{MHz}$ transducer applied parasternally to the shaved chest wall of mice anesthetized with pentobarbital, as previously described (19). In M-mode, the following parameters were obtained at the level of the papillary muscle: end-diastolic left ventricular internal diameter (LVIDd), end-systolic left ventricular internal diameter (LVIDs), and posterior wall and interventricular septum wall thickness. Fractional shortening (FS) was calculated as: ([LVIDd-LVIDs]/LVIDd) $\times 100$. Animals were subjected to serial weekly 
echocardiographic analyses after sham injection or EUK-8 treatment for the duration of the study (4 weeks).

\section{Statistical Analysis}

Data are expressed as means \pm SD. Differences among groups were tested by ANOVA. Comparisons between 2 groups were performed using the paired Student's t-test. $P<0.05$ was considered to be significant.

\section{Results}

Table 1 shows the body weight, heart weight, heart-to-body weight ratio, and echocardiographic findings in wild-type, EUK-8-treated wild-type, KO, and EUK-8-treated $\mathrm{KO}$ mice at 12 weeks of age. $\mathrm{KO}$ mice showed heart dilatation and a progressively increasing heart-to-body weight ratio compared to wild-type or EUK-8-treated wild-type mice. However, in EUK-8-treated KO mice, the heart weight and the heart-to-body weight ratio decreased compared to untreated $\mathrm{KO}$ mice.

To examine whether EUK-8 had preventive effects on cardiac dysfunction progression in $\mathrm{KO}$ mice, an echocardiographic study was performed. No significant differences in cardiac function exhibited as the percentage of fractional shortening were observed for all experimental mice with or without EUK-8 treatment. In contrast, KO mice showed an increased LV end-diastolic internal diameter (LVEDD) $(P<0.01)$, although no marked changes occurred in LVEDD of wild-type mice with or without EUK-8 treatment. Administration of EUK-8 for 4 weeks in KO mice decreased LV end-systolic internal diameter LVESD $(P<0.01)$ and LVEDD $(P<0.01)$, despite the normal cardiac function of 
KO mice with or without EUK-8 treatment. These results demonstrate that EUK-8 significantly prevented the progression of cardiac diameter caused by the loss of Mn-SOD.

Western blot analysis of Mn-SOD and catalase proteins in 12-week-old KO and wild-type mice with or without EUK-8 treatment is shown in Fig. 1. Protein extracts from heart, skeletal muscle, or liver were immunoblotted with anti-Mn-SOD, anti-catalase or anti-GAPDH. A specific loss of Mn-SOD expression in the heart and skeletal muscle of KO mice was observed, but no loss was observed in wild-type, EUK-8 treated wild-type and liver tissues of KO mice. However, treatment of KO mice with EUK-8 did not show the reduction of Mn-SOD or catalase expression. The present results therefore indicate that EUK-8 treatment inhibits the downregulation of expression for Mn-SOD or catalase in heart and skeletal muscle from mice.

In $\mathrm{KO}$ mice, H\&E staining showed that the LV wall exhibited myocardial degeneration and myocyte disarray (Fig. 2A,B). Masson's trichrome staining showed diffuse fibrotic scars surrounding the myocardial cells in $\mathrm{KO}$ mice. Some of the thickened fibrotic foci were likely caused by myocardial remodeling. These effects seen for $\mathrm{KO}$ mice were diminished in EUK-8 treated $\mathrm{KO}$ mice. To investigate whether Mn-SOD deficiency facilitated oxidative DNA damage in the heart, immunostaining of 8-oxodG, a biomarker of oxidative DNA damage, was examined. With DAPI staining, the signal intensities of nuclear DNA damage (red particles) significantly increased in $\mathrm{KO}$ mice heart tissue compared to that from wild-type mice (Figure 2C). Again, these intensities were significantly reduced in heart samples from EUK-treated KO mice. These results suggest that 
accumulated oxidative damage in nuclei was evident in $\mathrm{KO}$ mice, and that the antioxidant EUK-8 attenuated this damage $(p<0.01)$ (Figure 2D).

To examine the effect of MnSOD loss and EUK-8 treatment on cardiac telomeres, we examined telomere lengths from the different mouse groups. The mean telomere refraction fragment (TRF) was determined using a telomere percentage analysis. The fragment length was not significantly changed among heart tissues from wild-type, EUK-8-treated wild-type, KO, and EUK-8-treated KO mice. When each telomeric sample was divided into grid squares according to their molecular size, the results also did not differ among the four groups. The TRF data were similar for liver tissues as well, although the fragment length was decreased compared to heart tissue (Fig. 3 A,B).

To determine whether loss of MnSOD affected telomerase function, we quantified telomerase activity using a TRAP assay. Telomerase activity was found to be significantly decreased in heart tissues from $\mathrm{KO}$ mice, but not those from wild-type, EUK-8 treated wild-type and EUK-8-treated KO mice. The activity for liver tissue remained effectively unchanged among the four groups (Fig. 4). To further evaluate telomerase activity in the hearts of experimental mice, the catalytic subunits TERT, and the telomere-associated proteins TRF1 and TRF2 were assessed by Western blotting (Fig. 5). The protein expression of TERT was attenuated in KO mice, but not in wild-type, EUK-8 treated wild-type and EUK-8-treated KO mice. Similar results were obtained for TRF2 protein expression, while those of TERC and TRF1 were unchanged among these experimental groups (data not shown). The protein expression levels of these proteins were not different in liver tissue from the four groups. 
The telomerase activity of the catalytic subunits TERT and TRF2 were downregulated in hearts from KO mice and treatment with EUK-8 prevented those effects. To assess the regulation of cardiac muscle cell growth and survival in the MnSOD KO animal model, expression levels of cell signal transduction proteins were examined. Sirt1, FoxO3a, IGF-1, eNOS, phospho-AKt, p16, p27, and p53 were evaluated in hearts from each group by Western blot analysis (Fig. 6 A, B). Protein expression for IGF-1, eNOS, phospho-AKT and FoxO3a was significantly depressed in KO mice $(p<0.01)$, while the expression of Sirt1, p16, p27 and p53 was enhanced. These changes were abolished by treatment with EUK-8. These data indicate that the antioxidant treatment affected cell signaling not only for aging signals but also for survival factors.

\section{Discussion}

The present study showed that the synthetic antioxidant EUK-8 has preventive effects against oxidative DNA damage in heart/muscle-specific MnSOD-deficient mice having dilated LV chambers and depressed telomerase activity, which is likely due to reduced expression of the telomerase catalytic subunits TERT and TRF2 (22). Furthermore, in these KO mice, expression levels of proteins governing cell cycle, cell apoptosis, or cell survival were affected, while the expression of Sirt 1 , a protein involved in aging regulation $(23,24)$, was enhanced. These changes could be inhibited in KO mice by treatment with the antioxidant EUK-8.

Telomere attrition has attracted attention because of its association with 
cardiovascular disease (25) and has been shown to be related to an increased heart disease mortality rate. We observed no shortening of telomere length in the hearts from either wild-type or KO mice, or mice treated or untreated with EUK-8. This finding may be interpreted in several ways. The four week treatment beginning at 8 weeks of age may be too short, and a significantly longer observation period, such as several months, may be necessary to observe protective effects of antioxidants on telomere length. However, earlier studies with this animal model demonstrated that cardiac dilatation began at 8 weeks of age and there was a $50 \%$ cumulative survival rate by 14 weeks of age (5). In fact, relatively little is known about the physiological role of telomere length in healthy untreated WT C57/BI6 mice over time (21). So far, no shortening of telomeres has been reported between 3-week-old and 6-month-old $\mathrm{C} 57 / \mathrm{Bl} 6$ mice (26). It is therefore possible that the regulation of telomerase proteins in SOD KO mice-mediated survival signaling may be independent of telomere length.

Accumulation of oxidative damage is thought to play an important role in aging and associated diseases, including cardiovascular disease (27). Since most recent research has focused on the role of telomerase, it is of great importance to investigate whether alterations in additional telomere-associated proteins contribute to cardiovascular disease pathogenesis. In fact, prolonged oxidative damage inhibits telomerase activity and accelerates telomere shortening in VSMCs (28), which may be mediated by the formation of 8-oxodG at the GGG triplet in telomeric DNA (16). In isolated cells, TERT gene transfer reduces replicative senescence and extends the life span of numerous cell types, 
including cardiomyocytes (21).The aforementioned studies are consistent with the notion that oxidative stress promotes telomere exhaustion and decreases telomerase activity, perhaps by Src kinase family-dependent phosphorylation of hTERT at Try 707, which promotes nuclear export of telomerase (28). Haendeler et al. further demonstrated that reduction of intracellular ROS formation by treatment of endothelial cells with the antioxidant $\mathrm{N}$-acetylcysteine delays the loss of telomerase activity and the onset of age-related replicative senescence, by inhibiting nuclear export of telomerase (29). Here we observed that EUK-8 treatment of $\mathrm{KO}$ mice prevented the depressed telomerase activity and the reverse transcriptase responsible for the extension of telomeric repeat sequences, as well as decreases in TERT expression. Both telomerase and TERT have been shown to regulate cardiac muscle cell growth and survival (17), and their loss causes cardiac dysfunction, increased expression of p53, and increased apoptosis (18). The present study shows that EUK-8 suppresses oxidative DNA damage and thereby may increase telomerase activity in $\mathrm{KO}$ mice. Taken together, these results suggest that attenuation of oxidative damage improves impaired LV dilation and heart weight of $\mathrm{KO}$ mice despite there being no change in fractional shortening. Additionally, in vivo studies have shown that EUK-8 improves cardiac mitochondrial dysfunction $(7,8)$, delays aging $(30)$, and prevents adrenergic hypertrophy (31). Our results suggest that EUK-8 scavenges ROS generated in the cytoplasm or organelles, including mitochondria, because EUK-8 possesses both SOD and catalase activity and is a potent SOD/catalase mimetic and antioxidant $(7,8)$. EUK-8 also is able to reduce the amount of the oxidative DNA damage marker 8-oxodG, which is 
increased in the nuclear DNA from KO mice heart tissue.

The expression of Sirt 1 was increased in the hearts of KO mice, and this increase could again be attenuated by EUK treatment. Expression of Sirt1 was upregulated two-fold in response to stresses in KO mice (Fig. 6). This modest upregulation is likely to be a compensatory mechanism that may retard aging and inhibit apoptosis without causing mitochondrial dysfunction or NAD-depletion (31). Expression of Sirt1 was also upregulated in failing hearts (24) and in animals subjected to caloric restriction (23), suggesting that stimulation of known longevity mechanisms could be a new modality of heart failure treatment that increases resistance of heart tissue to pathologic insults. The anti-aging and stress-resistance effects of Sirt1 could be mediated not only by well-established mechanisms of longevity such as FoxO family transcription factors, but also by nonspecific upregulation of cytoprotective pathways (32). Thus, increased Sirt1 may protect the heart from oxidative stress by facilitating expression of antioxidants such as catalase by FoxO-dependent mechanisms (33), since Sirt1 expression is controlled by the transcription factors p53 and FOXO3a.

Our data show that antioxidant EUK-8 upregulates telomere-stabilizing proteins in the heart. The underlying mechanism is related to increased levels of IGF-1, phospho-eNOS, and phospho-Akt, which synergize with the activation of telomerase to protect against cellular senescent and apoptotic signaling events. These data are now extended by the observed effects on the regulation of telomere-associated protein TERT. In accordance with these observations, expression levels of TERT are increased, and might regulate survival as 
antiapoptotic factors (17). EUK-8 treatment also upregulates the expression of TRF2, which has been suggested to serve as a binding platform for additional telomere-associated proteins that mediate signal transduction to DNA damage checkpoint controls (26). TRF2 also mediates proapoptotic signaling in cardiomyocytes (22). In agreement with previous studies, it seems likely that TRF2 serves as a regulator of cellular aging and function beyond and potentially independently of telomere length protection (29).

Our data emphasize the role of mitochondrial ROS in cardiac aging, which is an independent predictor of mortality in a mouse longevity cohort. In conclusion, the administration of EUK-8 significantly prevented the depression of telomerase activity associated with its protein subunits as well as the morphological changes in an animal model of dilated cardiomyopathy, $\mathrm{H} / \mathrm{M}-\mathrm{SOD} 2^{-/}$. These results imply that telomerase activity may play a pivotal role as an antioxidant defense mechanism.

\section{ACKNOWLEDGMENTS}

We would like to thank Ms. K. Tsuchida, Ms. S. Taguchi, and Ms. Y. Ueda for their expert technical assistance. This work was supported in part by a Grant-in-Aid from the Ministry of Education, Science, and Culture of Japan.

DISCLOSURES; No conflicts of interest are declared by the author(s). References

1. Giordano FJ. Oxygen, oxidative stress, hypoxia, and heart failure. J Clin 
Invest 2005; 115:500-8.

2. Maier $\mathrm{CM}$, Chan $\mathrm{PH}$. Role of superoxide dismutases in oxidative damage and neurodegenerative disorders. Neuroscientist 2002; 8:323 - 334 .

3. Li Y, Huang TT, Carlson EJ, Melov S, Ursell PC, Olson JL, et al. Dilated cardiomyopathy and neonatal lethality in mutant mice lacking manganese superoxide dismutase. Nat Genet 1995; 11: 376 -81.

4. Lebovitz RM, Zhang H, Vogel H, Cartwright J Jr, Dionne L, Lu N, et al. Neurodegeneration, myocardial injury, and perinatal death in mitochondrial superoxide dismutase-deficient mice. Proc Natl Acad Sci USA 1996; 93:9782 87.

5. Nojiri H, Shimizu T, Funakoshi M, Yamaguchi O, Zhou H, Kawakami S, et al. Oxidative stress causes heart failure with impaired mitochondrial respiration. $J$ Biol Chem 2006; 281:33789 - 801.

6. Valenti L, Conte D, Piperno A, Dongiovanni P, Fracanzani AL, Fraquelli M, et al. The mitochondrial superoxide dismutase A16V polymorphism in the cardiomyopathy associated with hereditary haemochromatosis. J Med Genet $2004 ; 41: 946-50$.

7. van Empel VP, Bertrand AT, van Oort RJ, van der Nagel R, Engelen 
M, van Rijen HV, et al. EUK-8, a superoxide dismutase and catalase reduces cardiac oxidative stress and ameliorates pressure overload-induced heart failure in the harlequin mouse mutant. J Am Coll Cardiol 2006; 48:824-32.

8. Kawakami S, Matsuda A, Sunagawa T, Noda Y, Kaneko T, Tahara S, Hiraumi Y, Adachi S, Matsui H, Ando K, Fujita T, Maruyama N, Shirasawa T, Shimizu T. Antioxidant, EUK-8, prevents murine dilated cardiomyopathy. Circ J 2009; 73: 2125-34

9. Morten KJ, Ackrell BA, Melov S. Mitochondrial reactive oxygen species in mice lacking superoxide dismutase 2: Attenuation via antioxidant treatment. $\mathrm{J}$ Biol Chem 2008; 281:3354-9.

10. Xu Y, Armstrong SJ, Arenas IA, Pehowich DJ, Davidge ST. Cardioprotection by chronic estrogen or superoxide dismutase mimetic treatment in the aged female rat. Am J Physiol Heart Circ Physiol 2004; 287: H165 -71.

11. Melov S, Ravenscroft J, Malik S, Gill MS, Walker DW, Clayton PE, et al. Extension of life-span with superoxide dismutase/catalase mimetics. Science $2000 ; 289: 1567-9$.

12. Melov S, Doctrow SR, Schneider JA, Haberson J, Patel M, Coskun PE, et al. Lifespan extension and rescue of spongiform encephalopathy in superoxide dismutase 2 nullizygous mice treated with superoxide dismutase-catalase 
mimetics. J Neurosci 2001; 21:8348 -5353.

13. van Steensel B, Smogorzewska A, de Lange T. TRF2 protects human telomeres from end-to-end fusions. Cell. 1998; 92:401-13.

14. von Zglinicki T. Role of oxidative stress in telomere length regulation and replicative senescence. Ann N Y Acad Sci 2000; 908: 99-110.

15. Chang E, Hartley CB. Telomere length and replicative aging in human vascular tissues. Proc Natl Acad Sci 1995; 92:11190-119.

16. Kawanishi S, Oikawa S. Mechanism of telomere shortening by oxidative stress. Ann N Y Acad Sci. 2004; 1019: 278-84.

17. Oh H, Taffet GE, Youker KA, et al. Telomerase reverse transcriptase promotes cardiac muscle cell proliferation, hypertrophy, and survival. Proc Natl Acad Sci U S A 2001; 98:10308 -13.

18. Leri A, Franco S, Zacheo A, Barlucchi L, Chimenti S, Limana F et al. Ablation of telomerase and telomere loss leads to cardiac dilatation and heart failure associated with p53 upregulation. EMBO J 2003; 22:131-9.

19. Makino N, Naeda T, Oyama J, Higuchi Y, Mimori K. Improving insulin sensitivity via activation of PPAR-y increases telomerase activity in the heart of 
OLETF rats. Am J Physiol Heart Circ Physiol 2009; 297:H2188-96.

20. Maeda T, Guan JZ, Oyama J, Higuchi Y, Makino N. Aging-associated alteration of subtelomeric methylation in Parkinson's disease. J Gerontol A Biol Sci Med Sci. 2009; 64:949-55.

21. Danial NN, Korsmeyer SJ. Cell death: critical control points. Cell 2004; 116 : 205-21.

22. Fuster JJ, Andres V. Telomere biology and cardiovascular disease. Circ Res. 2006; 99:1167-80.

23. Sinclair DA. Toward a unified theory of caloric restriction and longevity regulation. Mech Ageing Dev. 2005; 126:987-1002.

24. Alcendor RR, Kirshenbaum LA, Imai S, Sadoshima J. Sir2, a longevity factor and a class III histone deacetylase, is an essential endogenous inhibitor of apoptosis in cardiac myocytes. Circ Res. 2004; 65:971-80.

25. Samani NJ, Boultby R, Butler R, Thompson JR, Goodall AH. Telomere shortening in atherosclerosis. Lancet 2002; 358:472-3.

26. Werner C, Hanhoun M, Widmann T, Kazakov A, Semenov A, Pöss J, et al. Effects of Physical Exercise on Myocardial Telomere-Regulating Proteins, 
Survival Pathways, and Apoptosis. J Am College of Cardiol 2008; 52:470-82.

27. Griendling KK, FitzGerald GA. Oxidative stress and cardiovascular injury. Part I: Basic mechanisms and in vivo monitoring of ROS. Circulation 2003; 108:1912-6.

28. Haendeler J, Hoffmann J, Brandes RP, Zeiher AM, Dimmeler S. Hydrogen peroxide triggers nuclear export of telomerase reverse transcriptase via Src kinase family-dependent phosphorylation of tyrosine. Mol Cell Biol. 2003; 23:4598-4610.

29. Haendeler J, Hoffmann J, Diehl JF, Vasa M, Spyridopoulos I, Zeiher AM, Dimmeler S. Antioxidants inhibit nuclear export of telomerase reverse transcriptase and delay replicative senescence of endothelial cells. Circ Res. $2004 ; 94: 768-75$.

30. Blasco MA, Funk W, Villeponteau B, Greider CW. Functional characterization and developmental regulation of mouse telomerase RNA. Science. 1995; 269:1267-1270.

31. Alcendor R, Gao S, Zhai P, Zablocki D, Holle E, Yu X, et al. Sirt1 regulates aging and resistance to oxidative stress in the heart . Circ Res 2007; 100: $1512-21$ 
32. Kops GJ, Dansen TB, Polderman P, Saarloos I, Wirtz K, Coffer P et al. Forkhead transcription factor FOXO3a protects quiescent cells from oxidative stress. Nature. 2002; 419:316-21.

33. Alcendor RR, Gao S, Zhai P, Zablocki D, Holle E, Yu X et al. Sirt1 Regulates Aging and Resistance to Oxidative Stress in the Heart. Circ Res. 100(2007) pp1512-1521

\section{FIGURE LEGENDS}

Figure 1. Western blot analysis of Mn-SOD, and catalase expressed in heart, skeletal muscle, and liver tissue from saline-treated wild-type (W), EUK-8-treated WT (W+EUK), H/M-Sod2 ${ }^{-/}$(KO), and EUK-8-treated KO (KO+EUK) mice at 12 weeks of age. EUK-8 was administered to $\mathrm{W}$ or KO mice from 8 to 12 weeks of age. Representative data are shown in panel $A$ and summarized results are shown in panel B. The open, gray, solid, or diagonal fill patterns indicate $\mathrm{W}, \mathrm{W}+\mathrm{EUK}, \mathrm{KO}$, and $\mathrm{KO}+\mathrm{EUK}$, respectively. GAPDH was used as an internal control. Each bar indicates the mean + SD (n=6). ${ }^{\text {a }} ;<0.05$ vs. wild-type, ${ }^{b} ; p<0.05$ vs.untreated KO mice

Figure 2. Histological samples from saline-treated wild-type (W), EUK-8-treated WT (W+EUK-8), H/M-Sod2 ${ }^{-/-}(\mathrm{KO})$, and EUK-8-treated KO mice (KO+EUK-8) at 
12 weeks of age. Each picture shows LV cross-sectional views with H\&E staining (A), Masson's trichrome staining (B), and 8-oxodG staining as a marker of oxidant stress (D). The positions of nuclei are determined by DAPI stain (blue). Original magnification $\times 400$. The summarized 8-oxodG signal intensities of DNA damage (D) are presented using the same fill pattern used in Figure 1 (mean \pm $\mathrm{SD}, \mathrm{n}=6){ }^{\mathrm{a}} ;<0.05$ vs. wild-type, ${ }^{\mathrm{b}} ; \mathrm{p}<0.05$ vs.untreated KO mice

Figure 3. Telomere length analysis by Southern blot using the mean telomere refraction fragment (TRF) method. Representative data from heart (open column) and liver (solid column) tissues from saline-treated wild-type (W), EUK-8-treated W (W+EUK), H/M-Sod2-/- $(\mathrm{KO})$, and EUK-8-treated KO $(K O+E U K)$ mice at 12 weeks of age (A). The summary from six experiments is shown (B). Open and closed bars show results from heart and liver, respectively. Values are mean $\pm S D(n=5)$.

Figure 4. Telomerase activity by the telomerase repeat amplification protocol (TRAP) method in heart and liver tissues from saline-treated wild-type (W), EUK-8-treated W, H/M-Sod2 ${ }^{-/-}$(KO), and EUK-8-treated KO mice at 12 weeks of age. Representative data are shown in panel A and summarized results are shown in panel B. The fill pattern is the same as that for Figure 1. Values are mean \pm SD of 5 experiments. ${ }^{\mathrm{a}} ;<0.05$ vs. wild-type, ${ }^{\mathrm{b}} ; \mathrm{p}<0.05$ vs. untreated KO mice, NC: negative control; PC: positive control.

Figure 5. Western blot analysis of telomere-associated proteins TERT, TERC, 
and TRF-2 from the hearts of saline-treated wild-type (W), EUK-8-treated W, $\mathrm{H} / \mathrm{M}-\mathrm{Sod}_{2}{ }^{--}(\mathrm{KO})$, and EUK-8-treated KO mice at 12 weeks of age.

Representative data are shown in panel A and summarized results are shown in panel $B$. The fill pattern is the same as that for Figure 1. Values are mean $\pm S D$ of 5 experiments. ${ }^{a} ;<0.05$ vs. wild-type, ${ }^{b} ; p<0.05$ vs. untreated KO mice

Figure 6. Western blot analysis for Sirt1, FOXO3a, IGF-1, eNOS, phospho-AKt, and cell-cycle inhibitors, p16, p27, p53, from the hearts of saline-treated wild-type (W), EUK-8-treated W, H/M-Sod2 ${ }^{-/}(\mathrm{KO})$, and EUK-8-treated KO mice at 12 weeks of age. Representative data are shown in panel $A$ and summarized results are shown in panel B. All data were quantified by densitometry, and the levels were normalized to that of GAPDH. The fill pattern is the same as that for Figure 1. Values are mean $\pm S D(n=5){ }^{a} ;<0.05$ vs. wild-type, ${ }^{b} ; p<0.05$ vs.untreated $\mathrm{KO}$ mice 
Fig 1

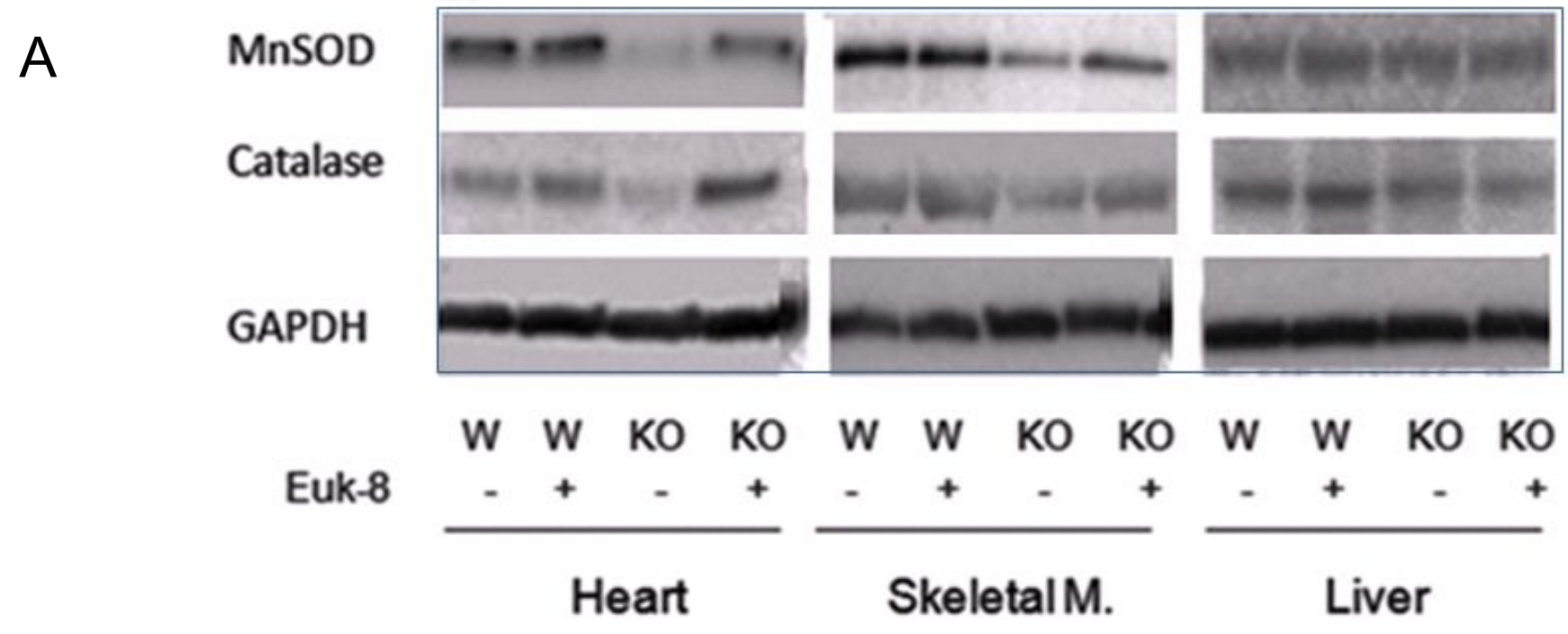

B
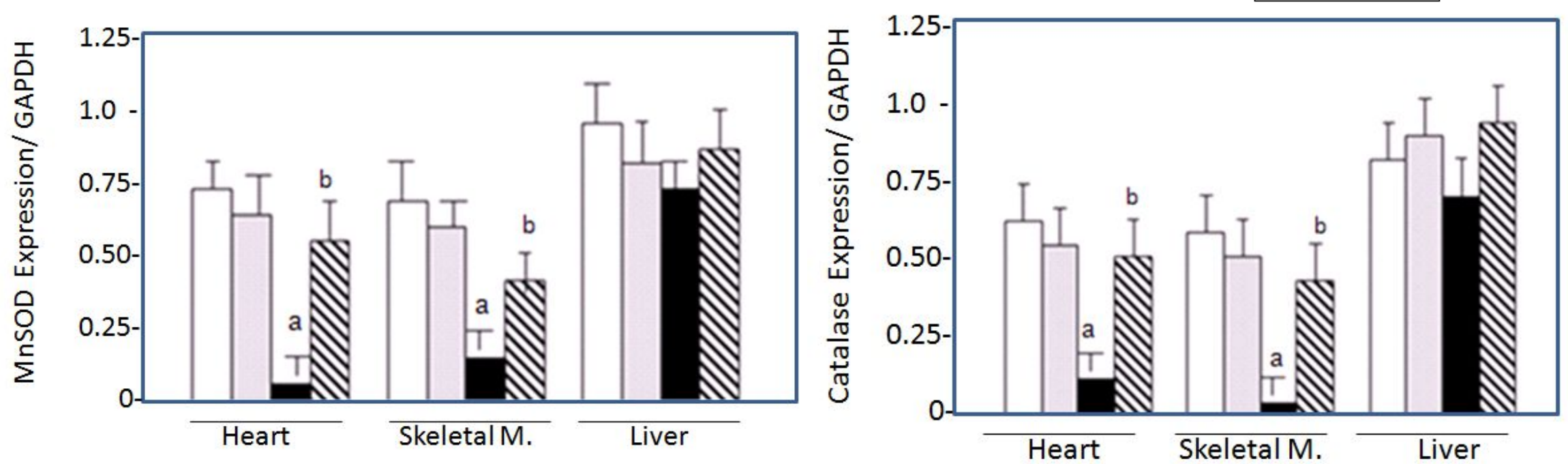
Fig-2

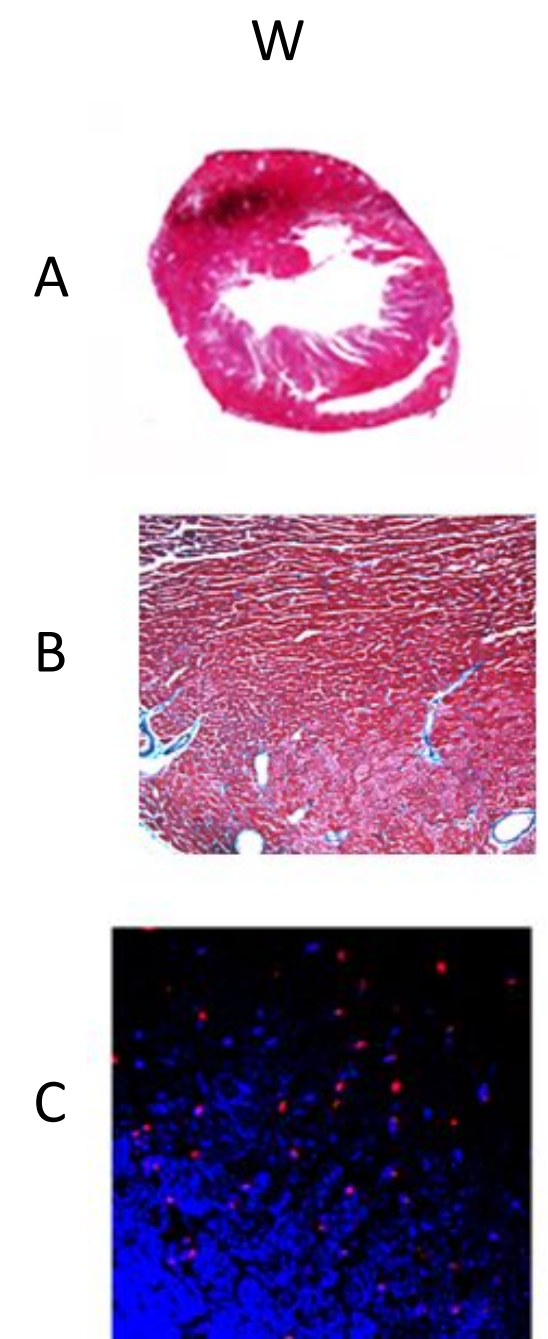

$$
W+E U K-8
$$

KO

KO+ EUK-8
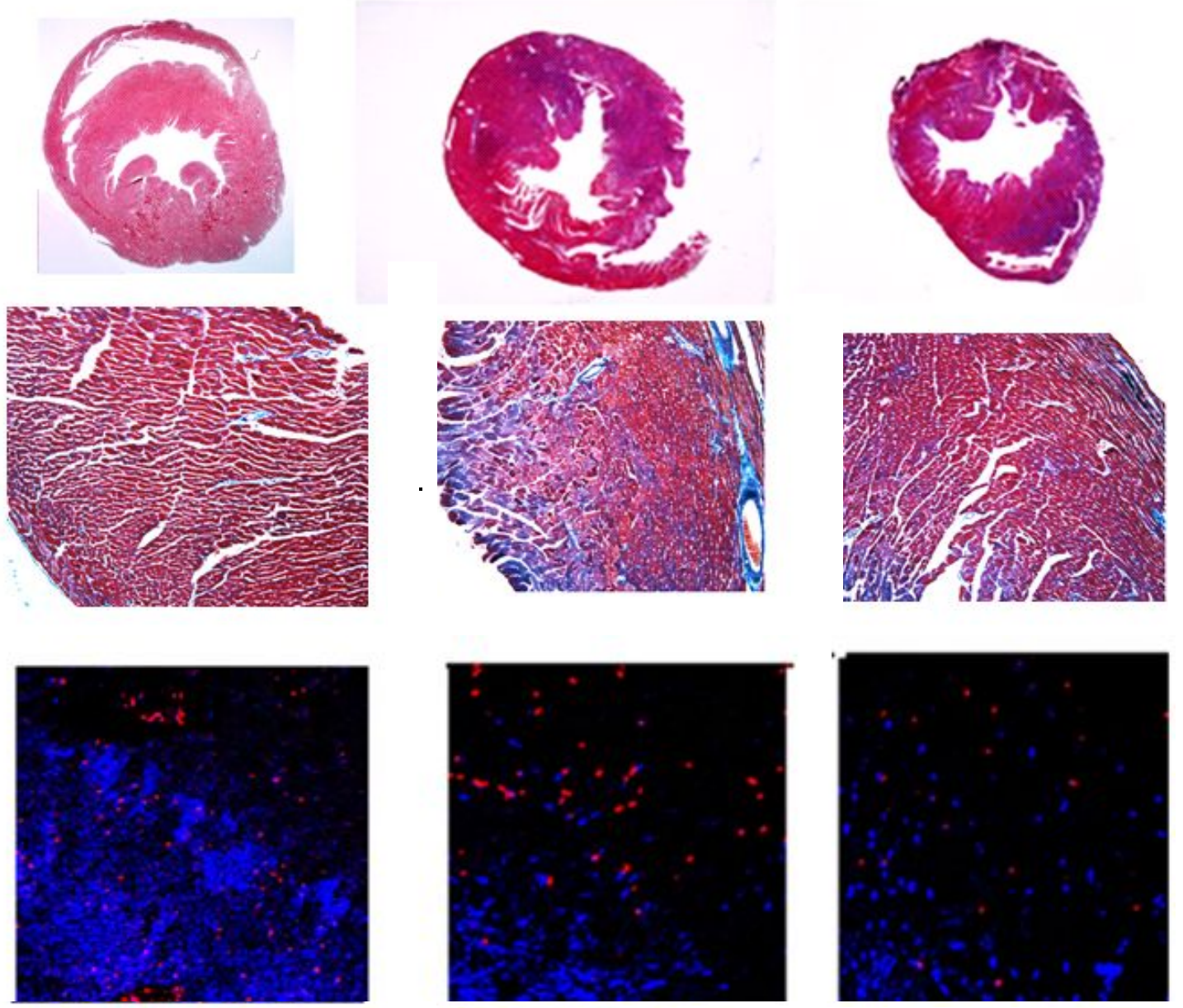


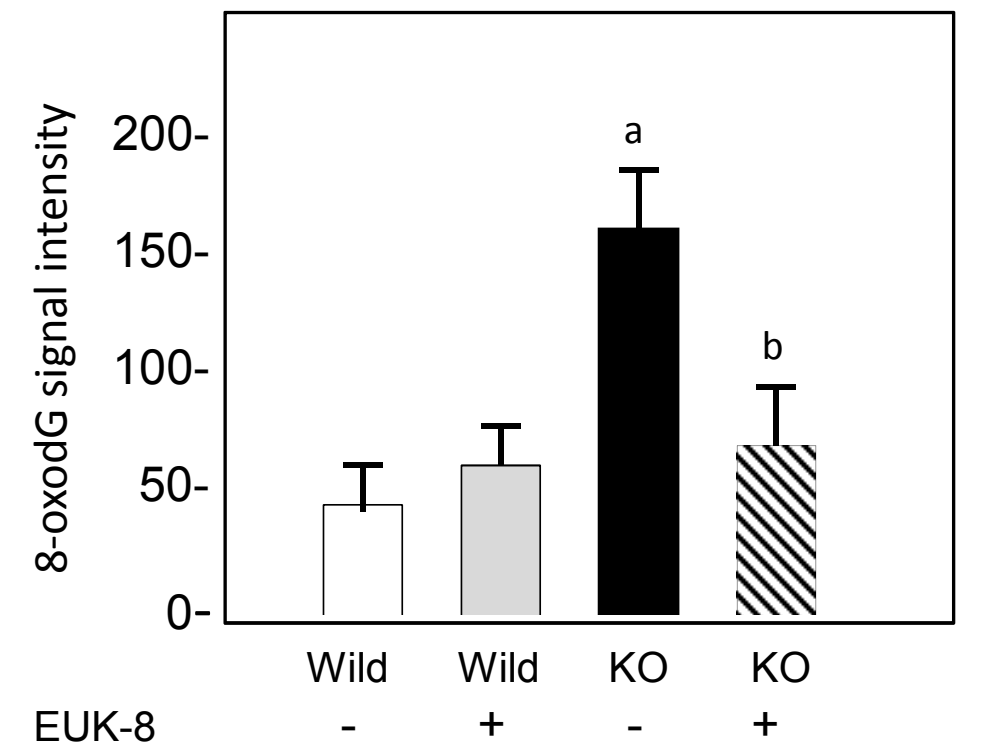


Fig-3

A

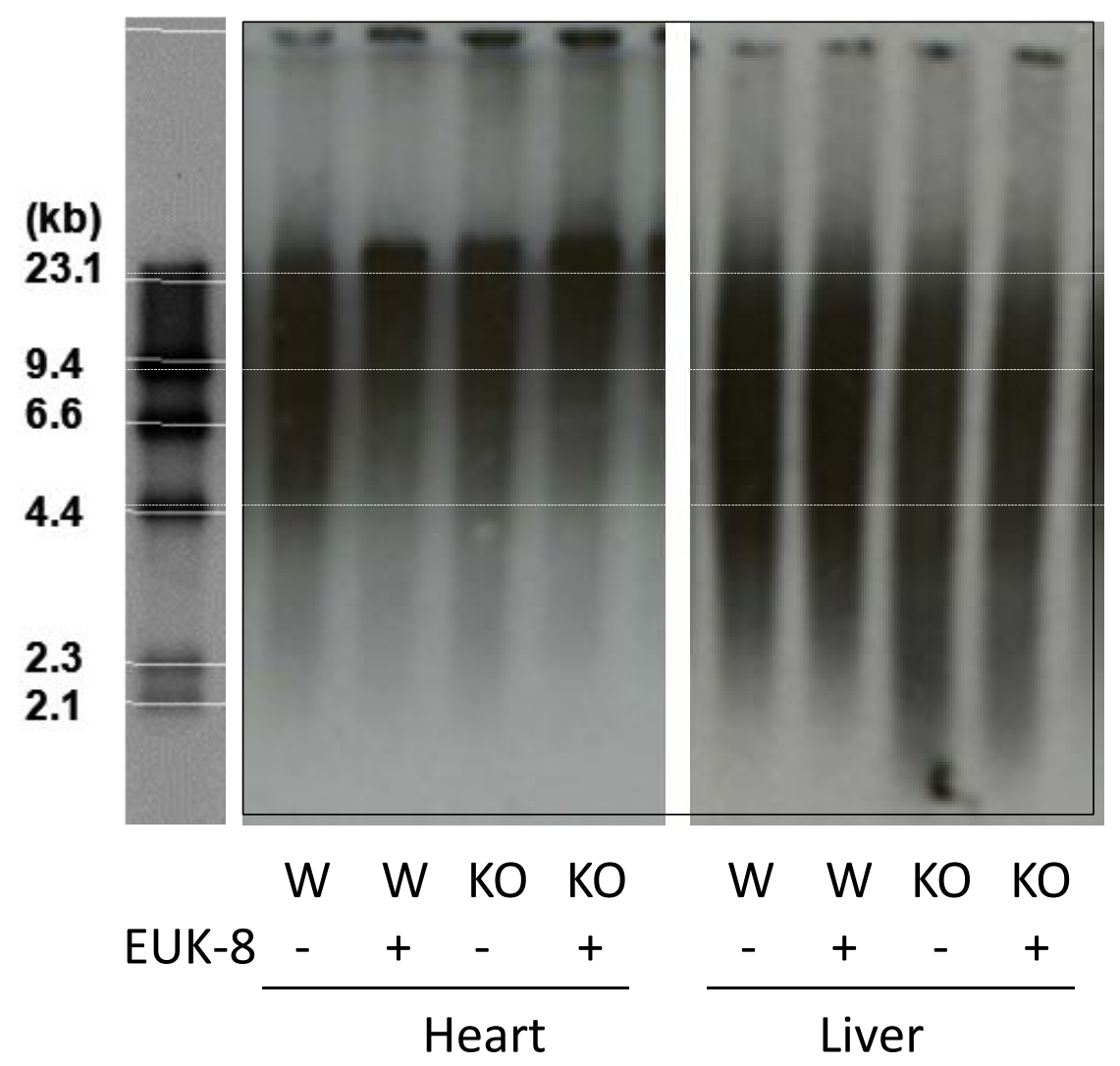

B

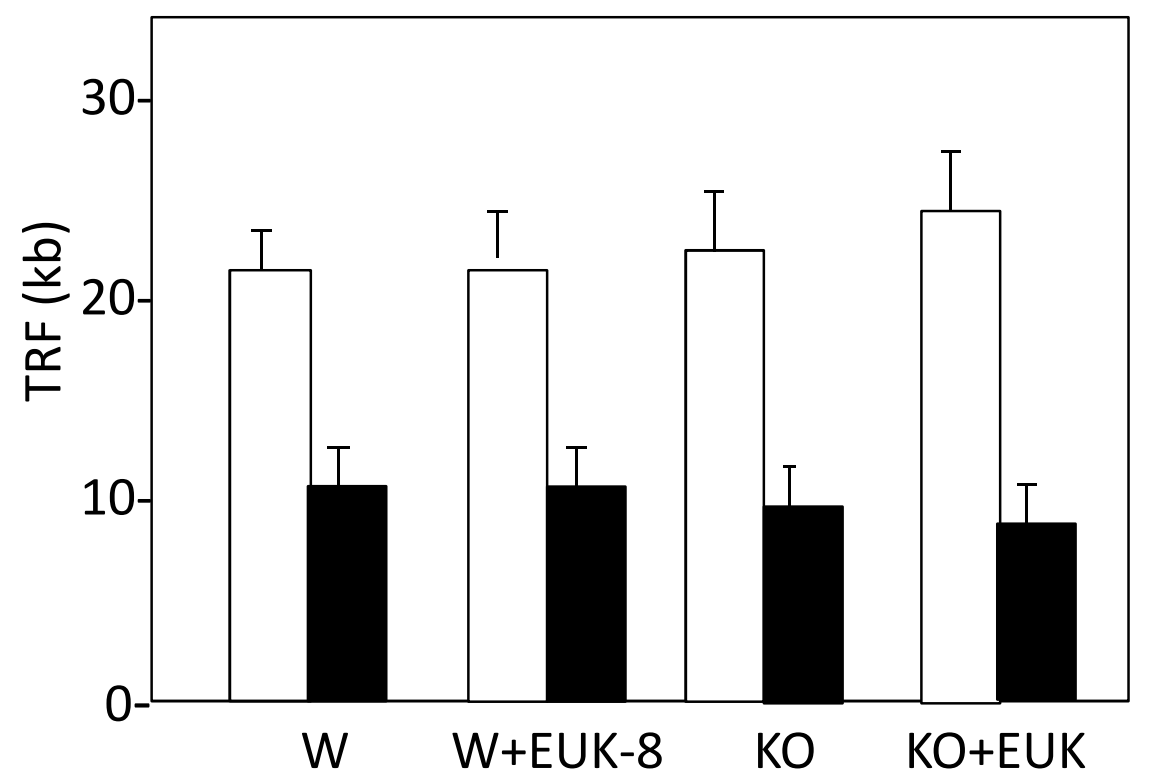


Fig 4

A
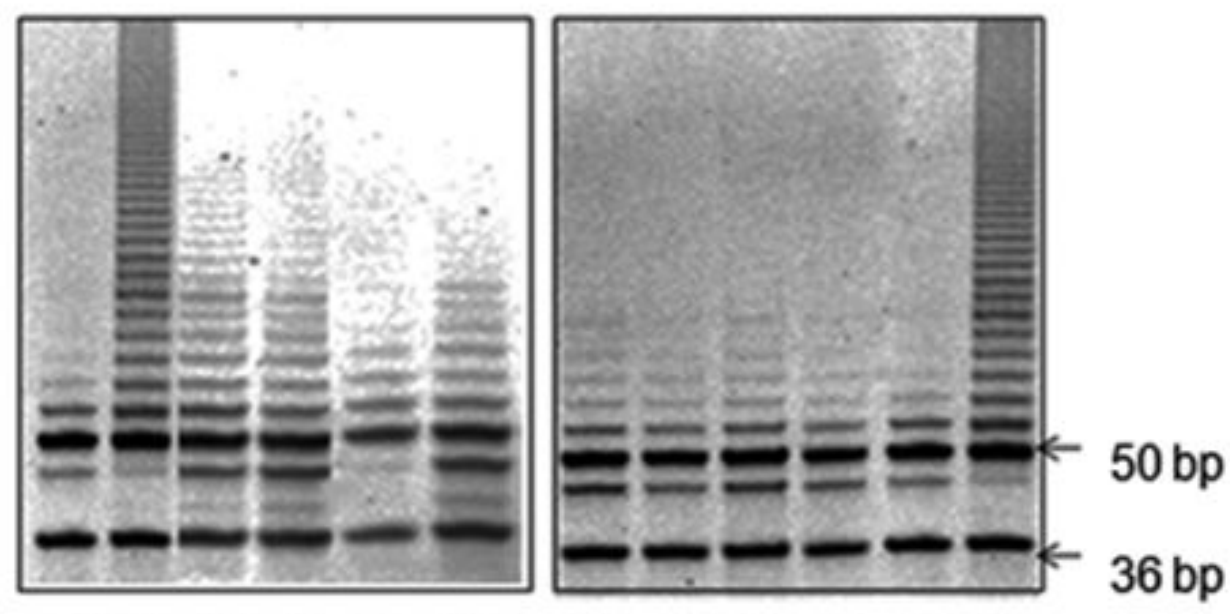

EUK-8

NC PC W W KO KO W W KO KO NC PC
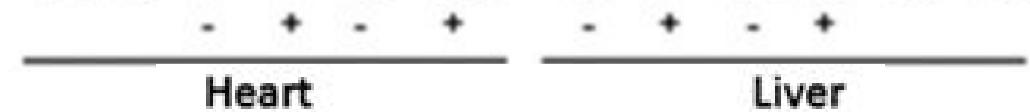

B

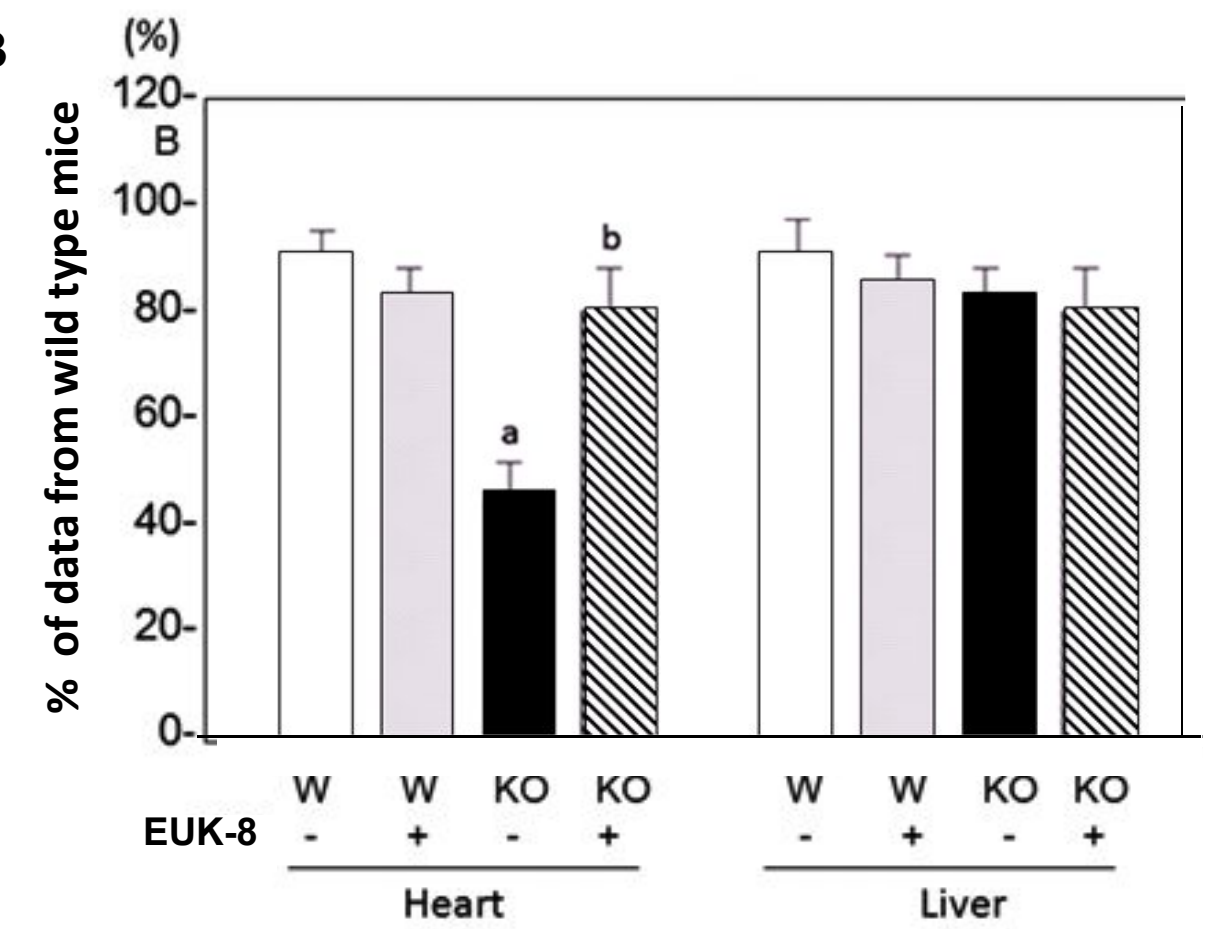


Fig 5

A

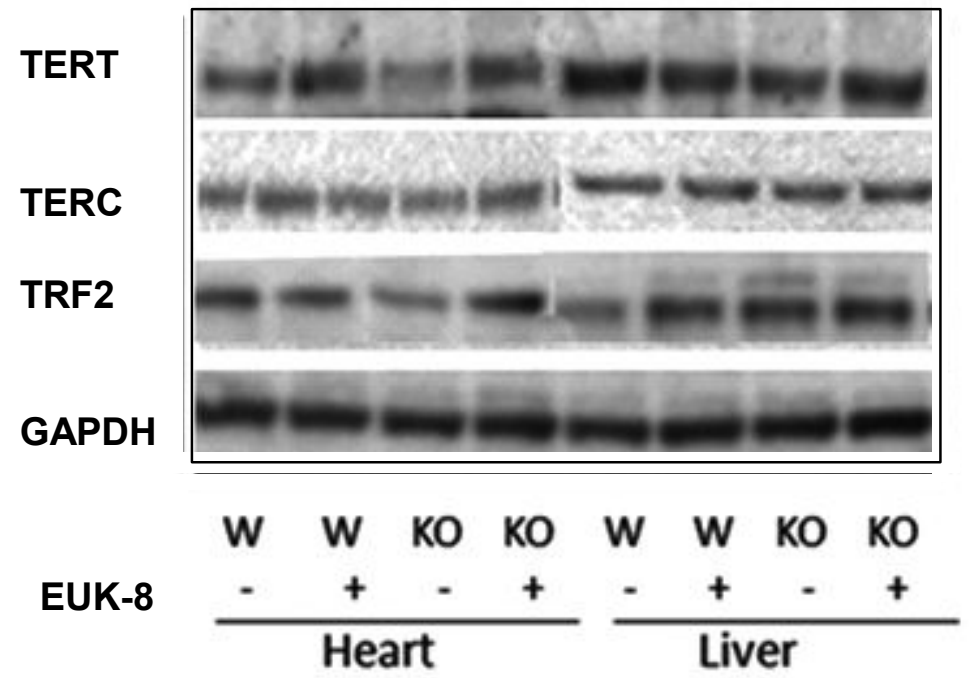

B

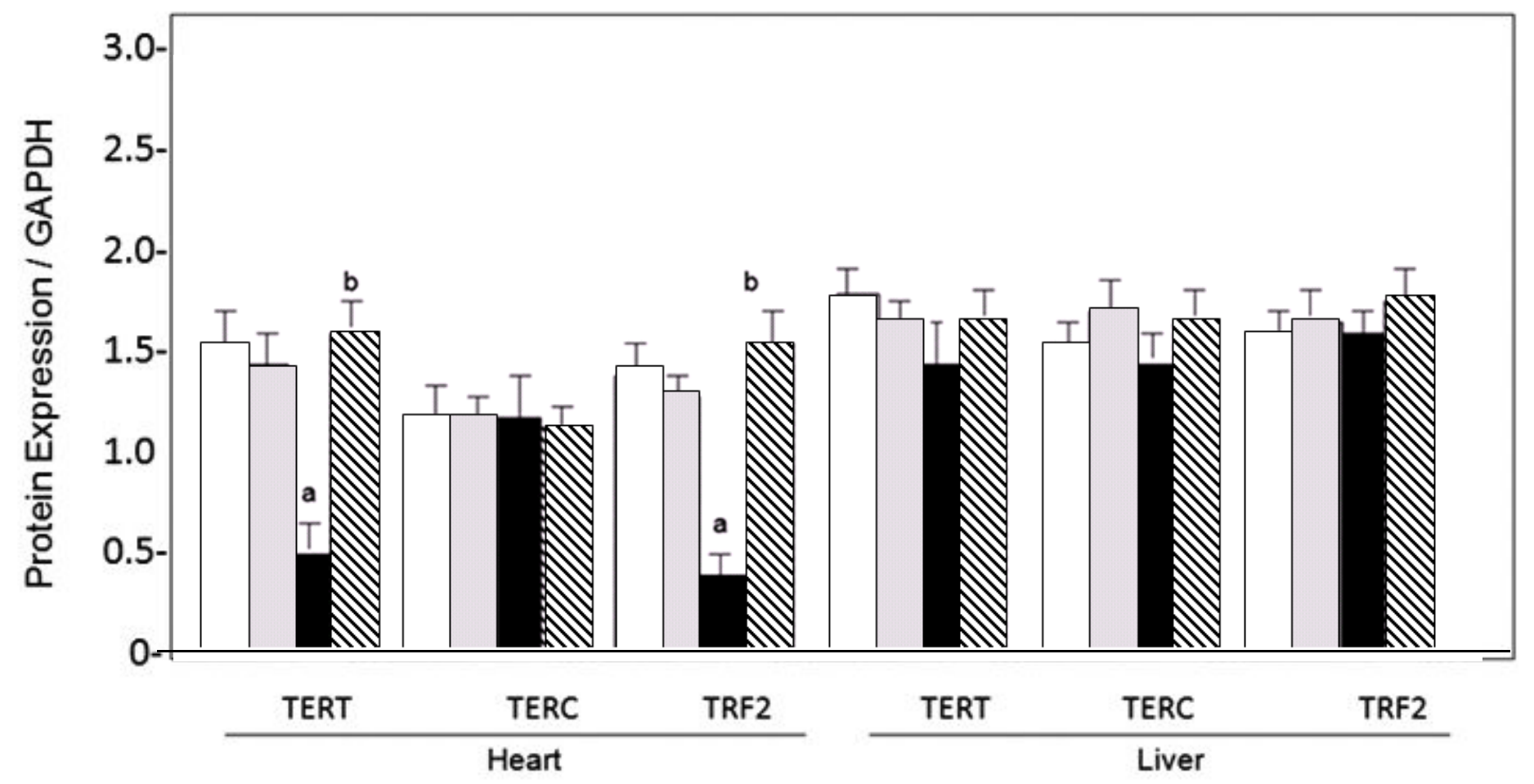


Fig 6
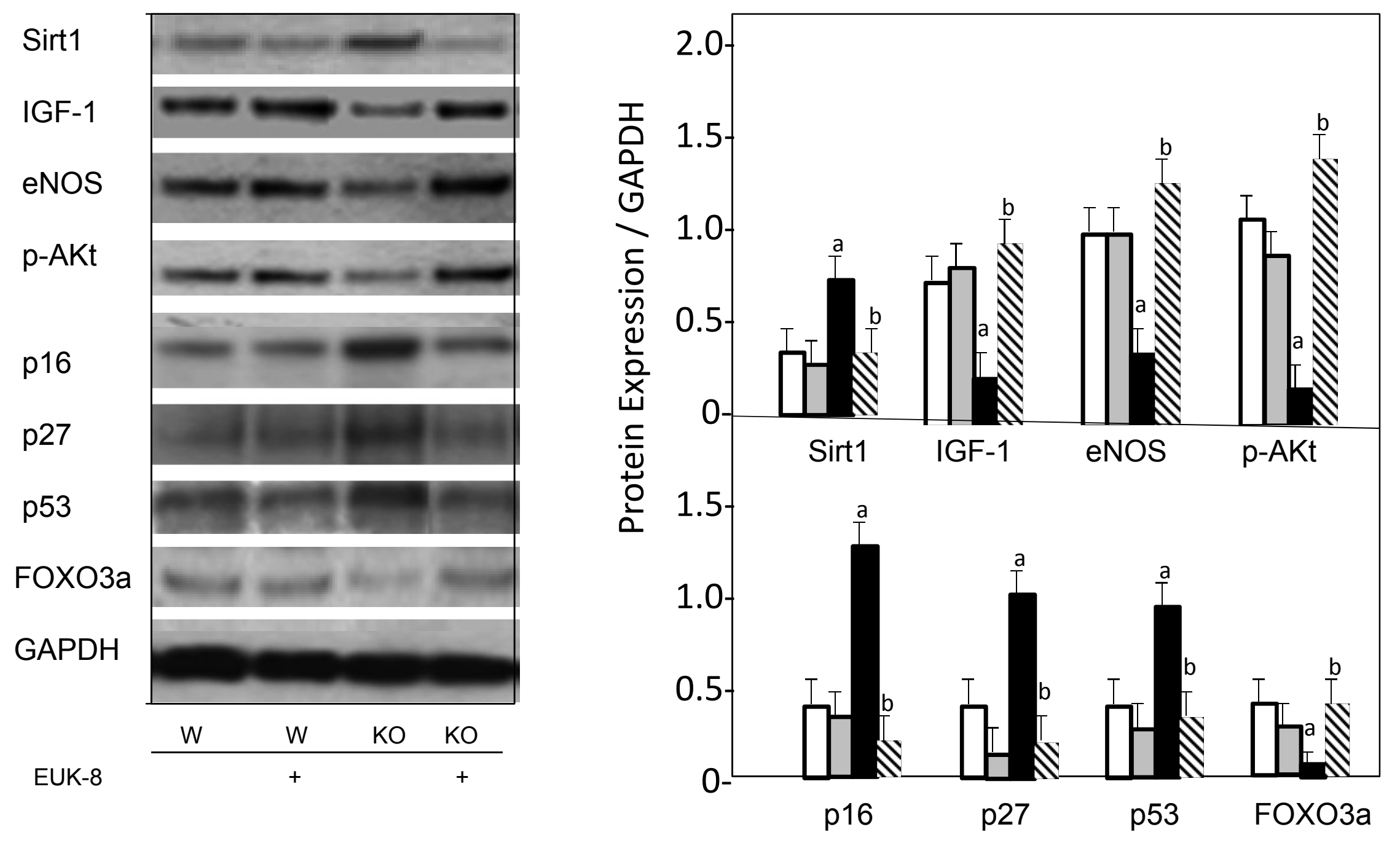
Table 1 Heart to body weight ratio and echocardiographic findings for 12 week old wild-type, EUK-8 treated wild-type, SOD KO and EUK-8 treated KO mice"

\begin{tabular}{|c|c|c|c|c|}
\hline & $\begin{array}{l}\text { Wild-type } \\
(n=6)\end{array}$ & $\begin{array}{l}\text { Wild-type + EUK-8 } \\
\qquad(n=6)\end{array}$ & $\begin{array}{l}\text { SOD KO } \\
(n=6)\end{array}$ & $\begin{array}{l}\text { SOD KO+ EUK-8 } \\
\qquad(n=6)\end{array}$ \\
\hline Body Wt. (g) & $26.7 \pm 2.1$ & $24.6 \pm 0.9$ & $21.8 \pm 1.1^{a}$ & $24.7 \pm 1.6^{b}$ \\
\hline Heart wt.(mg) & $179 \pm 14$ & $175 . \pm 11$ & $265 \pm 1.7^{\mathrm{a}}$ & $228 \pm 1.8^{\mathrm{a}, \mathrm{b}}$ \\
\hline Heart wt./Body wt. $\left(10^{-3}\right)$ & $6.71 \pm 0.5$ & $7.11 \pm 0.6$ & $12.1 \pm 0.7^{a}$ & $9.3 \pm 0.4^{a, b}$ \\
\hline IVS (mm) & $0.71 \pm 0.07$ & $0.70 \pm 0.09$ & $0.81 \pm 0.07$ & $0.83 \pm 0.05$ \\
\hline LVPWth (mm) & $1.12 \pm 0.07$ & $1.12 \pm 0.08$ & $1.11 \pm 0.06$ & $0.97 \pm 0.06$ \\
\hline LVEDD (mm) & $3.25 \pm 0.16$ & $3.33 \pm 0.14$ & $4.04 \pm 0.28^{a}$ & $3.30 \pm 0.10^{b}$ \\
\hline LVESD (mm) & $1.42 \pm 0.08$ & $1.38 \pm 0.08$ & $1.51 \pm 0.09$ & $1.28 \pm 0.08^{\mathrm{b}}$ \\
\hline FS (\%) & $56 \pm 3.9$ & $58 \pm 4.1$ & $63 \pm 4.9$ & $61 \pm 4.6$ \\
\hline $\mathrm{HR}$, beats/min & $462 \pm 23$ & $468 \pm 20$ & $473 \pm 21$ & $456 \pm 25$ \\
\hline
\end{tabular}

